\section{NOTIFICATIONS}

ata in this Public Health Bulletin relate to Epiweeks 1 to 7 . Table 10 lists the number of returns made to Epidemiology and Health Services Evaluation Branch this year. Public Health Units report to Epidemiology and Health Services Evaluation Branch weekly.

\section{TABLE 10}

NUMBER OF RETURNS TO E\&HSEB THIS YEAR

\begin{tabular}{|llll|}
\hline PHU & Number & PHU & Number \\
\hline Central/Southern & & Illawarra & 3 \\
Sydney & 5 & Hunter & 3 \\
Eastern Sydney & 3 & North Coast & 5 \\
South Western & & New England & 6 \\
Sydney & 4 & Orana \& Far West & 6 \\
Western Sector & 6 & Central West & 5 \\
Northern Sydney & 6 & South-West & 6 \\
Central Coast & 2 & South-East & 6
\end{tabular}

\section{ARBOVIRAL INFECTION}

Despite the heavy rains this summer arboviral activity has been lower this year than for the same period in 1991. High attack rates have been reported in the Northern Territory, Queensland and Western Australia.

\section{FOODBORNE DISEASE}

Foodborne diseases account for 18 per cent of notifications received in 1992.

A meeting between members of Epidemiology Branch and Food Branch recommended that Food Branch coordinate all intelligence activity arising from the identification of enteric pathogens by the Institute of Clinical Pathology and Medical Research, National Salmonella Surveillance System, Division of Analytical Laboratories and the Institute of Medical and Veterinary Science. Epidemiology Branch, through Public Health Units, should be responsible for surveillance of foodborne diseases through the established notification system. Members of Food Branch and Public Health Units are encouraged to collaborate at the local level to monitor foodborne diseases.

\title{
TABLE 11
}

RESPONSE TO QUESTIONNAIRE ON

SURVEILLANCE OF NON-NOTIFIABLE STDS

\begin{tabular}{|c|c|c|c|c|c|c|c|c|c|c|c|c|c|c|}
\hline Condition & CSA/SSA & ESA & SWS & WSAIWEN & NSA & CCA & ILL & HUN & NCR & NER & OFR & CWR & SWR & SER \\
\hline $\begin{array}{l}\text { Sexual Health } \\
\text { Centre in Area } \\
\text { Health Service? }\end{array}$ & Yes & Yes & Yes & Yes & Yes & Yes & Yes & Yes & Yes & Yes & Yes & No & No & No \\
\hline $\begin{array}{l}\text { Notifiable STDS } \\
\text { being notified } \\
\text { from SHC? }\end{array}$ & No & No & Yes & No & No & No & No & No & No & No & Yes & - & - & - \\
\hline $\begin{array}{l}\text { PHU able } \\
\text { to provide } \\
\text { aggregate } \\
\text { data for non- } \\
\text { notifiable STDs? }\end{array}$ & Yes & Yes & - & No May & - & March & No & Yes & Mar/Apr & - & Yes & - & - & - \\
\hline $\begin{array}{l}\text { Type of } \\
\text { database in } \\
\text { use by Sexual } \\
\text { Health Centre }\end{array}$ & $\begin{array}{c}\text { Macintosh/ } \\
\text { OMNI }\end{array}$ & $\begin{array}{l}\text { Sydney } \\
\text { SHC } \\
\text { dBASE }\end{array}$ & CRS & $\begin{array}{l}\text { CRS } \\
\text { Own } \\
\text { dBASE }\end{array}$ & - & $\begin{array}{l}\text { Sydney } \\
\text { SHC } \\
\text { dBASE }\end{array}$ & CRS & CRS & $\begin{array}{l}\text { Sydney } \\
\text { SHC } \\
\text { dBASE }\end{array}$ & $\begin{array}{l}\text { EHSEB } \\
\text { IDDS }\end{array}$ & $\begin{array}{l}\text { Epi- } \\
\text { Info }\end{array}$ & - & - & - \\
\hline $\begin{array}{l}\text { PHU able to } \\
\text { supply line } \\
\text { data for non- } \\
\text { notifiable STDs } \\
\text { by JULY } 1\end{array}$ & Yes & Yes & Yes & Yes Yes & Yes & Yes & No & No & $?$ & Yes & Yes & - & - & - \\
\hline
\end{tabular}

\section{SURVEILLANCE OF STDS}

Table 11 summarises the responses of the Public Health Units (PHUs) to a questionnaire on surveillance of sexually transmitted diseases (STDs). Seven PHUs will soon be able to provide information on the number of the non-notifiable STDs occurring in their Areas or Regions. Eight of the PHUs foresaw no problems in supplying individual record detail by July 1992 .

Only two Sexual Health Centres (SHCs) are complying with Public Health Act requirements to notify syphilis.

In obtaining individual record data it is essential that PHUs are not confronted with an increased data entry load.
It is anticipated that most $\mathrm{SHC}$ s will supply the data to PHUs on floppy disk that can be read into IDSS. For SHCs with dBASE systems this is straightforward. Where CRS is used the transfer is more involved. To overcome this the Health Department will develop a program that can extract data from CRS in a form that can be imported into the Department's IDSS program.

It should be emphasised that this new data set relates only to non-notifiable diseases. The notifiable diseases syphilis and AIDS - should be reported to PHUs, as required by the Public Health Act 1991. Gonorrhoea and HIV are notified by laboratory. 


\section{TABLE 12}

INFECTIOUS DISEASE NOTIFICATIONS BY HEALTH AREA AND REGION FEBRUARY 1992

\begin{tabular}{|c|c|c|c|c|c|c|c|c|c|c|c|c|c|c|c|}
\hline CONDITION & CSA & SSA & ESA & sws & WSA & WEN & NSA & HUN & NCR & NER & OFR & SWR & SER & U/K & TOTAL \\
\hline AIDS & 1 & - & - & - & - & - & 3 & $\overline{-}$ & - & - & - & - & - & - & 4 \\
\hline viral infect & 1 & - & $\overline{3}$ & - & - & $\overline{1}$ & $\overline{-}$ & 1 & - & - & - & $\overline{-}$ & $\bar{z}$ & $\overline{-}$ & 1 \\
\hline $\begin{array}{l}\text { Foodborne illness (NOS) } \\
\text { Gonorrhoea }\end{array}$ & 1 & $\overline{-}$ & $\begin{array}{l}3 \\
1\end{array}$ & $\overline{-}$ & $\overline{-}$ & $\frac{1}{-}$ & $\overline{-}$ & $\overline{-}$ & $=$ & $\bar{z}$ & $=$ & $\overline{-}$ & $\overline{-}$ & $\bar{z}$ & $\begin{array}{l}3 \\
1\end{array}$ \\
\hline H. influenzae meningitis & - & - & - & - & $\overline{1}$ & 1 & 1 & - & 1 & - & - & $\overline{-}$ & 1 & $\overline{-}$ & $\begin{array}{l}4 \\
1\end{array}$ \\
\hline $\begin{array}{l}\text { H. influenzae infection (NOS) } \\
\text { Hepatitis A - acute viral }\end{array}$ & $\overline{3}$ & $\overline{1}$ & $\overline{-}$ & $\overline{-}$ & 1 & $\overline{-}$ & $\overline{-}$ & $\overline{1}$ & $\overline{-}$ & $\overline{1}$ & $\overline{-}$ & $\overline{-}$ & - & - & 6 \\
\hline Hepatitis B - chronic/carrier & $\overline{2}$ & $\overline{2}$ & - & $\overline{2}$ & $\overline{-}$ & $\overline{-}$ & $\overline{3}$ & 1 & $\bar{z}+$ & $\overline{2}$ & $\overline{-}$ & $\overline{-}$ & $\overline{-}$ & $\bar{z}$ & 11 \\
\hline $\begin{array}{l}\text { Hepatitis B - Unspecified } \\
\text { Hepatitis C-acute viral }\end{array}$ & 2 & $\overline{-}$ & - & 1 & $\bar{z}$ & - & $\overline{7}$ & $\overline{-}$ & - & $=$ & - & - & $\overline{1}$ & - & 1 \\
\hline Hepatitis C - Unspecified & 4 & 2 & $\overline{10}$ & $\overline{1}$ & $\begin{array}{l}3 \\
1\end{array}$ & $\bar{z}$ & 7 & 12 & - & $\overline{-}$ & $\begin{array}{l}- \\
-\end{array}$ & $\overline{-}$ & 1 & $\overline{15}$ & 29 \\
\hline $\begin{array}{l}\text { HIV } \\
\text { Malar }\end{array}$ & $\stackrel{5}{-}$ & $\underline{2}$ & 10 & 1 & - & - & 3 & 1 & - & $\overline{-}$ & $\overline{-}$ & $\overline{-}$ & $\overline{-}$ & 15 & $\begin{array}{c}30 \\
1\end{array}$ \\
\hline Measles & 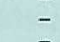 & - & - & - & - & 1 & 2 & 2 & - & 2 & $\overline{-}$ & $\overline{-}$ & $\overline{-}$ & - & $\frac{7}{2}$ \\
\hline Meningococcal infection (NOS) & - & $\overline{-}$ & - & - & $\overline{1}$ & 1 & $\stackrel{1}{1}$ & - & $\overline{-}$ & 1 & - & $\overline{-}$ & $\bar{z}$ & $\bar{z}$ & $\frac{2}{3}$ \\
\hline $\begin{array}{l}\text { Mycobacterial atypical } \\
\text { Mycobacterial tuberculosis }\end{array}$ & - & $\overline{-}$ & - & $=$ & 1 & 1 & 1 & $\overline{-}$ & - & $\overline{-}$ & - & - & z- & - & $\frac{3}{2}$ \\
\hline $\begin{array}{l}\text { Mycobacterlal tuderculosis } \\
\text { Pertussis }\end{array}$ & - & 1 & - & - & $\overline{-}$ & - & - & - & - & - & - & - & - & - & 1 \\
\hline $\begin{array}{l}\text { Rubella } \\
\text { Salmonella infection (NOS) }\end{array}$ & $\begin{array}{l}- \\
-\end{array}$ & - & $\overline{1}$ & $\overline{2}$ & $\begin{array}{c}1 \\
1\end{array}$ & - & I & - & $=$ & $\overline{1}$ & $\overline{1}$ & $\overline{1}$ & $\bar{z}$ & $\overline{-}$ & 7 \\
\hline $\begin{array}{l}\text { Salmonella infection (NOS) } \\
\text { Syphilis }\end{array}$ & 1 & - & 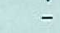 & 2 & . & - & 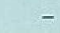 & 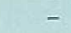 & 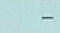 & 1 & - & - & - & - & 4 \\
\hline
\end{tabular}

\section{TABLE 13}

INFECTIOUS DISEASE NOTIFICATIONS

BY HEALTH AREA AND REGION

JANUARY 1 TO FEBRUARY 29, 1992

\begin{tabular}{|c|c|c|c|c|c|c|c|c|c|c|c|c|c|c|c|c|c|c|c|}
\hline CONDITION & CSA & SSA & ESA & sws & WSA & WEN & NSA & CCA & ILL & HUN & NCR & NER & OFR & CWR & SWR & SER & OTH & U/K & TOTAL \\
\hline Adverse event after immunisation & & - & - & - & - & 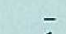 & - & - & - & - & 2 & 1 & 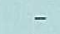 & - & 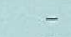 & 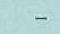 & - & - & \\
\hline AIDS & 3 & $\overline{-}$ & 3 & $\overline{-}$ & 1 & 1 & 3 & $\overline{-}$ & $\overline{-}$ & $\overline{2}$ & - & - & $\overline{1}$ & - & $\overline{1}$ & - & & $\overline{-}$ & 11 \\
\hline $\begin{array}{l}\text { Teetion } \\
\text { illness (NOS) }\end{array}$ & $\overline{1}$ & - & $1 \overline{8}$ & $\overline{1}$ & $1 \overline{6}$ & $\overline{3}$ & 1 & $\overline{5}$ & $\overline{-}$ & 3 & - & $\overline{2}$ & $\begin{array}{l}1 \\
2\end{array}$ & - & $\begin{array}{l}1 \\
1\end{array}$ & - & $\overline{-}$ & - & 52 \\
\hline Gastroenteritis (instit) & 4 & - & $1 \overline{0}$ & 2 & $\overline{-}$ & $\overline{-}$ & - & - & $\overline{2}$ & $\overline{1}$ & $\bar{z}$ & $\begin{array}{l}3 \\
1\end{array}$ & 1 & I & - & 2 & - & - & 23 \\
\hline H. influenzae epiglottitis & 4 & - & - & 2 & - & - & $\overline{-}$ & $\overline{-}$ & - & 1 & $\overline{2}$ & - & 1 & - & - & $=$ & - & & 3 \\
\hline $\begin{array}{l}\text { H. influenzae meningitis } \\
\text { H. influenzae septicaemia }\end{array}$ & - & 1 & - & - & $\overline{-}$ & 2 & 1 & - & - & 2 & $\frac{2}{1}$ & $\overline{-}$ & - & - & 1 & 1 & - & $\overline{-}$ & $\begin{array}{r}10 \\
1\end{array}$ \\
\hline H. influenzae infection (NOS) & 1 & & 1 & $\bar{z}$ & 1 & - & 1 & - & - & $\overline{0}$ & - & $-\overline{0}$ & $\overline{-}$ & - & $\overline{2}$ & 1 & 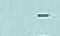 & - & 5 \\
\hline Hepatitis A - acute viral & 22 & 4 & 31 & 2 & 4 & 1 & 15 & - & 1 & -8 & 2 & 10 & $\begin{array}{l}1 \\
3\end{array}$ & - & -2 & 1 & - & $\overline{-}$ & $\begin{array}{r}104 \\
11\end{array}$ \\
\hline $\begin{array}{l}\text { Hepatitis B - acute viral } \\
\text { Hepatitis B - chronic/carrier }\end{array}$ & 1 & 1 & $\overline{-}$ & $\overline{1}$ & $\overline{7}$ & $\overline{2}$ & 6 & $\overline{-}$ & $\overline{-}$ & $\overline{9}$ & - & $\overline{1}$ & 1 & - & 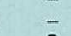 & 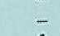 & 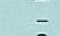 & - & 21 \\
\hline Hepatitis B - Unspecified & 30 & 16 & 7 & $\begin{array}{l}39 \\
14\end{array}$ & $\begin{array}{r}15 \\
4\end{array}$ & 1 & 30 & 1 & 2 & 5 & $\frac{2}{8}$ & 7 & 1 & 1 & 3 & 1 & 2 & $\overline{-}$ & $\begin{array}{r}163 \\
43\end{array}$ \\
\hline $\begin{array}{l}\text { Hepatitis } C \text { - acute viral } \\
\text { Hepatitis } C \text { - Unspecified }\end{array}$ & 26 & 11 & $2 \overline{6}$ & 6 & 12 & 1 & 17 & - & 2 & $4 \overline{6}$ & 12 & 5 & - & - & - & 2 & - & - & 166 \\
\hline $\begin{array}{l}\text { Hepatitis, acute viral (NOS) } \\
\text { HIV infection }\end{array}$ & 10 & 3 & 29 & 2 & 3 & 1 & 4 & 1 & - & 2 & 2 & - & 1 & - & - & 1 & - & 33 & \\
\hline & & & & - & 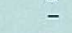 & - & & 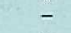 & & & 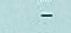 & 1 & . & 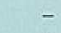 & - & $\overline{-}$ & & & \\
\hline ase & - & - & - & $\bar{z}-$ & $\bar{z}$ & 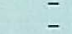 & 1 & - & - & $\begin{array}{l}- \\
-\end{array}$ & - & $\overline{-}$ & - & $\overline{3}$ & & 1 & & & \\
\hline $\begin{array}{l}\text { Leptospirosis } \\
\text { Malaria }\end{array}$ & - & - & - & 1 & 1 & 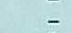 & 2 & $\overline{-}$ & - & - & $\overline{-}$ & $\overline{-}$ & - & 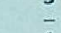 & - & 1 & - & & \\
\hline Measles & 3 & 4 & 3 & 7 & 1 & 2 & 3 & 3 & 2 & 11 & 4 & 3 & & 1 & & 2 & & & 49 \\
\hline gococcal septicaemia & - & $\overline{-}$ & - & 2 & $\overline{-}$ & - & $\overline{1}$ & - & 1 & - & - & 2 & - & E & - & $\bar{z}$ & z- & $\overline{-}$ & \\
\hline gococcal infection (NOS) & - & $\overline{-}$ & $\overline{-}$ & - & $=$ & - & - & $\overline{-}$ & 1 & $\overline{3}$ & - & 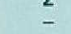 & - & - & - & - & - & & \\
\hline Mycobacterial atypical & 1 & 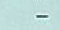 & 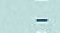 & 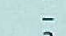 & 2 & 1 & 2 & - & & 1 & & 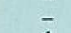 & 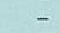 & 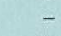 & 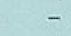 & $\overline{-}$ & - & & \\
\hline $\begin{array}{l}\text { Mycobacterial tuberculosis } \\
\text { Mycobacterial infection (NOS) }\end{array}$ & 1 & z & 2 & 3 & 4 & $\begin{array}{l}1 \\
1\end{array}$ & 4 & 1 & $\frac{1}{-}$ & $\bar{z}$ & 2 & $\frac{1}{-}$ & $\overline{1}$ & - & - & 1 & $\overline{-}$ & $\bar{z}$ & $\begin{array}{r}21 \\
2\end{array}$ \\
\hline $\begin{array}{l}\text { Mertussis } \\
\text { Pertal intection (NOS) }\end{array}$ & - & 3 & 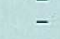 & 2 & 1 & 1 & - & - & - & - & 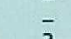 & $\overline{-}$ & & - & & - & & - & \\
\hline $\begin{array}{l}\text { Q feve } \\
\text { Rubel }\end{array}$ & - & $\bar{z}$ & $\bar{z}$ & - & 1 & $\bar{z}$ & $\overline{1}$ & $\overline{-}$ & $\overline{-}$ & 1 & 2 & 3 & & - & - & $\bar{z}$ & 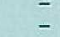 & $\bar{z}$ & \\
\hline ella infection (NOS) & 角 & 11 & 14 & 8 & 11 & 5 & 11 & - & - & 3 & 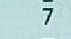 & 6 & 8 & 5 & 2 & 4 & 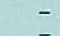 & - & 102 \\
\hline Sypt & 11 & 2 & 4 & 8 & 2 & - & 2 & $\overline{-}$ & $\bar{z}$ & 1 & & 2 & & - & & - & 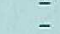 & $\bar{z}$ & 4I \\
\hline $\begin{array}{l}\text { Tetanus } \\
\text { Typhoid \& paratyphoid }\end{array}$ & $\overline{1}$ & $\overline{-}$ & - & - & $\overline{1}$ & $\bar{z}$ & $\overline{3}$ & $\overline{-}$ & $\overline{-}$ & $\overline{-}$ & $\overline{-}$ & $\overline{-}$ & $\overline{-}$ & - & - & - & - & - & 5 \\
\hline
\end{tabular}

Abbreviations used in this Bulletin:

CSA Central Sydney Health Area, SSA Southern Sydney Health Area, ESA Eastern Sydney Health Area, SWS South Western Sydney Health Area, WSA Western Sydney Health Area, WEN Wentworth Health Area, NSA Northern Sydney Health Area, CCA Central Coast Health Area, ILL Illawarra Health Area, HUN Hunter Health Area, NCR SER South East Health Region, OTH Interstate/Overseas, U/K Unknown, NOS Not Otherwise Stated

Please note that the data contained in this Bulletin are provisional and subject to change because of late reports or changes in case classification. Data are tabulated where possible by area of residence and by the disease onset date and not simply the date of notification or receipt of such notification. 